\title{
Review \\ Overdiagnosis and overtreatment of breast cancer Is overdiagnosis an issue for radiologists?
}

\section{Ruth Warren and Asha Eleti}

Department of Radiology, University of Cambridge, Cambridge, UK

Corresponding author: Ruth Warren, rmlw2@cam.ac.uk

Published: 21 April 2006

This article is online at http://breast-cancer-research.com/content/8/2/205 (c) 2006 BioMed Central Ltd
Breast Cancer Research 2006, 8:205 (doi:10.1186/bcr1396)

overdiagnosis, but in general when the radiologist finds a lesion labelled cancer by a pathologist, they and the patient regard this as a desirable event and the patient feels gratitude for a beneficial medical service. within the life of the patient and is one of the downsides of screening. This applies to low-grade ductal carcinoma in situ and some small grade 1 invasive cancers. Radiologists are responsible for cancer diagnosis, but at the time of diagnosis they cannot determine whether a particular low-grade diagnosis is one to which the definition of overdiagnosis applies. Overdiagnosis is likely to be driven by technological developments, including digital mammography, computer-aided detection and improved biopsy techniques. It is also driven by the patient's fear that cancer will be missed and the doctor's fear of litigation. It is therefore an issue of importance for radiologists, presenting them with difficult finetuned decisions in every assessment clinic that are ultimately counted later by those who evaluate their screening.

\section{Introduction}

Many of the previous entries in this review series have come from statisticians and epidemiologists who evaluate screening programmes and guide national policies for the health care of populations. Their calculations take into account the benefits for health and mortality, adverse consequences, cost-effectiveness and opportunity costs of obtaining evidence from trials and service screening [1]. They largely use data from events in the past to make forward decisions. Likewise, many of the pathological data are obtained by observing the different pathological entities in longitudinal follow up of case material stored many years ago and linked with outcomes. By contrast, radiologists, with the patient and the clinical teams, make current decisions for individuals.

Overdiagnosis is the result of detecting cancers that would not present in the patient's life without screening. Radiologists consider issues of overdiagnosis but they seldom use this particular term. Considerable radiological effort has been applied to reducing false-positive recalls, which result in unnecessary surgery for benign disease. Some of this literature is relevant to the present issue of
This situation is not unique to screening. In the current international political debate on pensions, if individuals could foresee their year of death and predict future fiscal performance then they would make good decisions on pension investment and age of retirement. This example has an individual and a population dimension, because current demographic changes were not foreseen when earlier policies were laid down. To use an example from a symptomatic clinical setting, a patient presenting with chest symptoms and an area of shadowing on a chest radiograph will wish to take an antibiotic for pneumonia, although the diagnosis is not conclusive, the disease may be of viral origin and many bacterial pneumonias resolve without treatment. The physician knows that this will avert the small possibility of death from pneumococcal pneumonia, a formerly lifethreatening disease that is now treatable.

\section{Small cancer nonspecific signs}

Radiological education for breast screening concentrates on the diagnosis of invasive cancers at small size when the tumour is fully treatable [2], because this is the situation in which screening has an impact on mortality for some women. As the point of diagnosis is moved ever earlier to smaller tumours, with radiological signs that tend to become less specific, more queries will concern benign and borderline lesions or lesions that turn out to be low-grade in situ or invasive tumours [3]. These are the lesions of the type found by chance in the breasts of women who die from other causes who are examined in the post mortem room [4]. Detection of these lesions while the patient is still alive results in overdiagnosis, which wastes resources and places patients at risk for overtreatment. These nonspecific signs fall 
broadly into two groups: small anonymous masses, in which the suspicion is small invasive cancer; and calcifications, which are more commonly associated with carcinoma in situ. This statement would justify a policy of greater vigilance for small masses than for calcifications. The distinction is not complete, however. There is good evidence that calcification may be associated with invasive tumours of aggressive type [5]; that a high proportion of high-grade ductal carcinoma in situ (DCIS) cases will progress to high-grade invasive cancers if they are left untreated [6]; that screening units with high DCIS detection rates also have high rates of detection of small invasive cancers [7]; and that assessed patients who later develop cancer at the assessed site frequently exhibited calcifications that with present policies would have been biopsied [8].

\section{Developing technology}

Since the original trials of breast screening conducted in the 1980s, there has been steady improvement in the associated technology. Development of screen-film technology, now to become superseded by digital mammography, has improved the detection of small cancers [9], both small masses and calcifications. This now has the potential to be enhanced by computer-aided detection [10,11]. Biopsy techniques have evolved with great sophistication, allowing tiny foci of nonspecific calcification to be sampled with great accuracy [12]. Lesions can be characterized further using contrastenhanced breast magnetic resonance imaging (MRI), which has great sensitivity for small invasive cancers in dense breasts [13]. MRI-guided biopsies are now showing that DCIS can also be detected at small size in dense breasts by contrast-enhanced MRI [14].

The availability of the techniques does not mandate their use, but there is a steady drift toward subjecting more and smaller lesions to biopsy, particularly because this can now be done percutaneously rather than by open surgical biopsy. A policy of aggressively biopsying calcification increases the rate of detection of DCISs, some of which will be of high grade with or without microinvasion or even of small high-grade invasive cancers. This is useful diagnostic activity. Less useful is the diagnosis of atypical hyperplasia and lobular carcinoma in situ, for which the best treatment is not well understood [15]. Excision biopsy is commonly carried out for such lesions in view of the possibility of progression to invasive cancer or the association with DCIS, and this may well constitute 'overtreatment'.

\section{Informed consent}

Women's understanding of the benefits and shortcomings of breast screening is imperfect [16]. Radiologists may be involved in writing the leaflets that inform women and advertisements that promote participation in screening. However, they are much more likely to be involved in one-toone verbal communications with individual women when they are informed of their mammograph findings and of the diagnostic and treatment decisions that ensue. They are likely to discuss the potential benefits and pitfalls of screening and the possibility that a biopsy procedure will progress to extensive surgery, depending on the subsequent pathological findings. Although they have a duty to be honest, it is unlikely that they will discuss the possibility of finding and treating a cancer that would not have presented in life, because they cannot prospectively distinguish such a case from one that does progress and requires treatment.

\section{Preferences of doctor and patient}

Both patients and the doctors who advise them have a range of attitudes to risk that are intrinsic to their personalities. Decisions will be a composite of the interaction of the two personalities. A doctor who is heavily risk averse is likely to advise a patient to proceed to biopsy and treatment of any suspect lesion, and many patients take the view that they wish no stone to be left unturned to ensure that they do not have cancer. The policies of a screening unit will represent the consensus of the attitudes of the participant radiologists, backed by procedures for policy made at national level. The UK National Health Service Breast Screening Programme has a policy document for assessment procedures [17]. Although a document of this type is designed to provide guidance, it can become the benchmark by which individual practitioners and their decisions are judged. This promotes more intensive biopsying of radiologically indeterminate lesions in a drive to improve cancer detection but at the cost of overdiagnosis.

\section{Litigation}

Screening carries with it the implicit problem that some cancers will not be diagnosed by screening, and it has been shown that in any screening programme a small proportion of these cancers will be visible on hindsight [18]. Inevitably, this results in dissatisfaction because patients feel that the screening programme, and more specifically the radiologist, has let them down, and should be called to account. Some of these women will pursue their claim through the courts. This environment creates a culture in which no stone is left unturned [19]. The doctor fears the accusation of missing a cancer and so will err on the side of caution and risk overdiagnosis. Few patients will complain that too much was done, but one of the authors of this review (RW) recently had litigation pending (finally dropped before going to court) on account of calcification not biopsied but later shown to be benign in the field of an emergent breast cancer. Concurrently, another patient made a major complaint about surgical 'mutilation' resulting from removal of an area of atypical ductal hyperplasia. Complaints and litigation are a minefield in which one realizes that individual patients have widely differing attitudes to priorities for their care.

\section{The effect of age}

Thus far the discussion has referred to population screening of women at standard levels of risk and classical age for 
mammographic screening (say $45-75$ years). Age and familial risk level will affect the incidence of cancer (invasive and noninvasive) in the screened population. Costs and benefits will differ, and so may the probability of overdiagnosis. When screening women who are younger than 45 years, some of the points made above will not apply. The detection of DCIS, whatever the grade, may be expected to be worthwhile because there are many more potential years of life for these lesions to progress from in situ to invasive disease. Similarly, low-grade invasive cancer may well have time to affect the woman's health. Cancers diagnosed in women under 45 years old have a greater probability of being of high grade than in older women [2]. On the other hand, the background incidence of cancer is lower, so proportionally the downsides of screening, including overdiagnosis, are of greater magnitude.

For older women (those older than 75 years), both the incidence and the detection of cancer are high, so the probability of a small tubular carcinoma or low-grade DCIS being found that would never present clinically is greater. Other fatal diseases become more common at this age, and it is these intercurrent diseases that increase the risk for overdiagnosis, because more women will die from other causes before their screen-detected cancer would become a problem [20].

\section{High-risk groups}

For high-risk groups the calculations will be different. For women with germline mutations in breast cancer susceptibility genes, particularly $B R C A$, the cancers are likely to be of high grade, and the discovery of DCIS is infrequent [21]. Overdiagnosis is unlikely to occur, and all suspect findings should be pursued to full diagnosis and treatment. This would be the wish of such high-risk patients, but it is also logical, irrespective of age. The biology of cancers in women who received mantle radiotherapy for lymphoma in adolescence may differ from those related to germline mutations, but again the incidence of cancer in this group will justify a high level of caution with any lesion. The psychological reaction of women with higher levels of risk has not been fully evaluated and may not necessarily be the same as that of women who are at standard risk. A recent systematic review identified this as a research priority [22].

\section{Overdiagnosis and treatment decisions}

Radiologists in screening programmes provide the role of diagnostic physicians working with surgeons and oncologists in the multidisciplinary team. They have a duty in view of their understanding of cancer to use their influence to bring sanity to treatment decisions for minimal cancers. In such cases, these teams must consider whether the treatment options should be used conservatively: axillary clearance would be overtreatment for a small tubular carcinoma; mastectomy would be an excessive surgical choice for a small area of lowgrade DCIS; and radiotherapy, which may potentially affect cardiac or pulmonary performance, may not be needed for
This article is part of a review series on

Overdiagnosis and overtreatment of breast cancer, edited by Nick E Day, Stephen Duffy and Eugenio Paci.

Other articles in the series can be found online at http://breast-cancer-research.com/articles/ review-series.asp?series=BCR_Overdiagnosis

small low-grade invasive tumours or low-grade DCIS. These women need a balanced approach to their treatment, which takes into account the concept that screening is a procedure driven by health policy and is different from disease of symptomatic presentation. Patients may need protection from their doctors, and fully informed consent is essential to the individual woman's understanding of her disease.

\section{Conclusion}

Radiologists are the physicians who implement the breast screening policies proposed by public health physicians, epidemiologists and health service managers. They are the ones who make the overdiagnosis happen, but they do this in ignorance of the evolution of the disease that they diagnose in each individual woman. When the patient asks, 'Will my disease kill me?' or 'Will my disease return after treatment?', the radiologist can only reply with probabilities and cannot tell the patient what will happen to them as an individual. Overdiagnosis is therefore a difficult issue for radiologists. It sits in the pan of a balance that has litigation and missed cancer in the other pan, and is as much a matter of patient wellbeing. It is therefore an issue of importance for radiologists, presenting them with difficult fine-tuned decisions in every assessment clinic that are ultimately counted later by those who evaluate their screening.

\section{Competing interests}

The authors declare that they have no competing interests.

\section{References}

1. Moss SM: Overdiagnosis and over treatment of breast cancer: overdiagnosis in randomised controlled trials of breast cancer screening. Breast Cancer Res 2005, 7:230-234.

2. Tabar L, Duffy SW, Vitak B, Chen HH, Prevost TC: The natural history of breast carcinoma: what have we learned from screening? Cancer 1999, 86:449-462.

3. Taplin SH, Ichikawa LE, Kerlikowske K, Ernster VL, Rosenberg RD, Yankaskas BC, Carney PA, Geller BM, Urban N, Dignan MB, et al:: Concordance of breast imaging reporting and data system assessments and management recommendations in screening mammography. Radiology 2002, 222:529-535.

4. Welch $\mathrm{H}$, Black $W$ : Using autopsy series to estimate the disease "reservoir" for ductal carcinoma in situ of breast: how much more breast cancer can we find? Ann Intern Med 1997, 127:1023-1028.

5. Tabar L, Tony Chen HH, Amy Yen MF, Tot T, Tung TH, Chen LS, Chiu YH, Duffy SW, Smith RA: Mammographic tumor features can predict long-term outcomes reliably in women with 1-14$\mathrm{mm}$ invasive breast carcinoma. Cancer 2004, 101:1745-1759.

6. Erbas B, Provenzano E, Armes J, Gertig D: The natural history of ductal carcinoma in situ of the breast: a review. Breast Cancer Res Treat 2005 [Epub ahead of print]. 
7. Evans A, Blanks R: Should breast screening programmes limit their detection of ductal carcinoma in situ? Clin Radiol 2002, 57:1086-1089.

8. Warren R, Allgood P, Hunnam G, Godward S, Duffy S: An audit of assessment procedures in women who develop breast cancer after a negative result. J Med Screen 2004, 11:180186.

9. Pisano ED, Gatsonis C, Hendrick E, Yaffe M, Baum JK, Acharyya S, Conant EF, Fajardo LL, Bassett L, D'Orsi C, et al.: Diagnostic performance of digital versus film mammography for breastcancer screening. N Engl J Med 2005, 353:1773-1783.

10. Birdwell R, Bandodkar P, Ikeda D: Computer-aided detection with screening mammography in a university hospital setting. Radiology 2005, 236:451-457.

11. Khoo L, Taylor P, Given-Wilson R: Computer-aided detection in the United Kingdom National Breast Screening Programme: prospective study. Radiology 2005, 237:444-449.

12. Heywang-Kobrunner S, Schreer I, Decker T, Bocker W: Interdisciplinary consensus on the use and technique of vacuumassisted stereotactic breast biopsy. Eur J Radiol 2003, 47: 232-236.

13. Buchanan C, Morris E, Dorn P, Borgen P, Van Zee K: Utility of breast magnetic resonance imaging in patients with occult primary breast cancer. Ann Surg Oncol 2005, 12:1045-1053.

14. Viehweg $P$, Bernerth $T$, Kiechle M, Buchmann J, Heinig A, Koelbl $\mathrm{H}$, Laniado M, Heywang-Kobrunner SH: MR-guided intervention in women with a family history of breast cancer. Eur J Radiol 2006, 57:81-89.

15. Sneige N, Lim S, Whitman G, Krishnamurthy S, Sahin A, Smith T, Stelling CB: Atypical ductal hyperplasia diagnosis by directional vacuum-assisted stereotactic biopsy of breast microcalcifications. Considerations for surgical excision. Am J Clin Pathol 2003, 119:248-253.

16. Domenighetti G, D'Avanzo B, Egger M, Berrino F, Perneger T, Mosconi $P$, Zwahlen M: Women's perception of the benefits of mammography screening: population-based survey in four countries. Int J Epidemiol 2003, 32:816-821.

17. Clinical guidelines for breast cancer screening assessment [http://www.cancerscreening.nhs.uk/breastscreen/publications/ assessment.html]

18. Hofvind S, Skaane $P$, Vitak B, Wang $H$, Thoresen S, Eriksen L, Bjorndal $H$, Braaten A, Bjurstam N: Influence of review design on percentages of missed interval breast cancers: retrospective study of interval cancers in a population-based screening program. Radiology 2005, 237:437-443.

19. Elmore JG, Taplin SH, Barlow WE, Cutter GR, D'Orsi CJ, Hendrick RE, Abraham LA, Fosse JS, Carney PA: Does litigation influence medical practice? The influence of community radiologists' medical malpractice perceptions and experience on screening mammography. Radiology 2005, 236:37-46.

20. Mandelblatt J, Saha S, Teutsch S, Hoerger T, Siu AL, Atkins D, Klein J, Helfand M; Cost Work Group of the U.S. Preventive Services Task Force: The cost-effectiveness of screening mammography beyond age 65 years: a systematic review for the U.S. Preventive Services Task Force. Ann Intern Med 2003, 139:835-842.

21. Lakhani SR, Jacquemier J, Sloane JP, Gusterson BA, Anderson TJ, van de Vijver MJ, Farid LM, Venter D, Antoniou A, Storfer-Isser $\mathrm{A}$, et al:: Multifactorial analysis of differences between sporadic breast cancers and cancers involving BRCA1 and BRCA2 mutations. J Nat/ Cancer Inst 1998, 90:1138-1145.

22. Watson E, Henderson B, Brett J, Bankhead C, Austoker J: The psychological impact of mammographic screening on women with a family history of breast cancer: a systematic review. Psychooncology 2005, 14:939-948. 